\title{
Analyses of antigenic types of Orientia tsutsugamushi in naturally infected Leptotrombidium colonies by improved immunofluorescent microscopy using monoclonal antibodies
}

\author{
Hiroshi Urakami*,1), 2), Koji Окuво ${ }^{2), 3)}$, Masahiro Fukuhara ${ }^{2)}$, \\ Hitoko MisUmi ${ }^{4)}$ and Mamoru TAKAHASHI ${ }^{4)}$ \\ * Corresponding author: Food Safety, Faculty of Applied Life Sciences, Niigata University of Pharmacy and Applied \\ Life Sciences (NUPALS), 265-1 Higashijima, Akiha-ku, Niigata 956-8603, Japan (E-mail: urakami@nupals.ac.jp) \\ ${ }^{1)}$ Food Safety, Faculty of Applied Life Sciences, Niigata University of Pharmacy and Applied Life Sciences (NUPALS), \\ 265-1 Higashijima, Akiha-ku, Niigata 956-8603, Japan \\ ${ }^{2)}$ Microbiology, Faculty of Pharmacy, Niigata University of Pharmacy and Applied Life Sciences (NUPALS), \\ 265-1 Higashijima, Akiha-ku, Niigata 956-8603, Japan \\ ${ }^{3)}$ Echigo Medical, 3-2 Koshoji, Nagaoka, Niigata 940-2106, Japan \\ 4) Department of Anesthesiology, Saitama Medical University, \\ Moroyama-machi, Iruma-gun, Saitama 350-0451, Japan
}

(Received: 4 November 2013; Accepted: 20 March 2014)

\begin{abstract}
Antigenic types of Orientia tsutsugamushi in naturally infected colonies of Leptotrombidium (L.) fletcheri, L. arenicola, and L. pallidum were examined using ten monoclonal antibodies specific to the type-specific antigen proteins of prototype strains (Gilliam, Karp, Kato, Kawasaki and Kuroki) by improved immunofluorescent microscopy. All antibodies were used at a 1:1,000 dilution, at which the antibodies reacted specifically to only one of the prototypes used for production of the antibodies. O. tsutsugamushi in L. fletcheri reacted to three of four anti-Kato antibodies, however only one antibody reacted to O. tsutsugamushi in all individual L. fletcheri, and two antibodies reacted only to the microorganisms in some of the mites. Similar results were obtained with anti-Karp antibodies to L. arenicola and L. pallidum. Experiments with serially diluted antibodies indicated that the fluctuation occurred only with antibodies, the end-points of which were close to $1: 1,000$. Therefore, our observations suggested that a single mite was not infected with multiple strains and that each infected colony of Leptotrombidium harbored a single antigenic strain of $O$. tsutsugamushi that is antigenically similar but different in some degree from the prototype strains.
\end{abstract}

Key words: Orientia tsutsugamushi, Leptotrombidium, antigenic type, transovarial transmission, monoclonal antibody

\section{INTRODUCTION}

Scrub typhus is a febrile disease caused by Orientia tsutsugamushi, which belongs to Rickettsiaceae, a family consisting of obligate intracellular parasitic bacteria, so-called rickettsiae. O. tsutsugamushi is transmitted to humans by chigger mites, such as Leptotrombidium pallidum (Nagayo, Miyagawa, Mitamura and Tamiya) in Japan and L. fletcheri (Womersly and Heaslip) in Southeast Asia. The rickettsiae are transovarially transmitted from female parents to their progeny in the mites (Rapmund et al., 1969).

Antigenic diversity among the strains is one of the characteristics of $O$. tsutsugamushi. The strains of $O$. tsutsugamushi are classified into several antigenic prototypes by reactivity of polyclonal (Elisberg et al., 1968) and monoclonal antibodies (Murata et al., 1986). Shirai et al. $(1979,1981,1982)$ analyzed the antigenicity of O. tsutsugamushi in the chigger mites and human patients by immunofluorescence microscopy (IF) using type-specific hyperimmune sera, and suggested that many of the individual mites and human patients were infected simultaneously with several strains of different antigenicity. They also indicated that the infected larval mites often harbored antigenic strains that had not been found in their female parents in spite of the fact that rickettsiae are transmitted only from the female parents (Shirai et al. 1982).

The antigenicity of this microorganism largely depends on the type-specific antigen (TSA) protein located on the surface of the microorganism (Tamura et al., 1985, Murata et al., 1986). Analyses by using monoclonal antibodies against the TSA proteins indicated that each O. tsutsugamushi strain isolated from an individual patient had only a single antigenicity (Ohashi et al., 1996). Ree et al. (1997) sequenced the TSA genes of $O$. tsutsugamushi in the mites and showed a single sequence of the TSA gene in a single mite. Although these reports did not agree with Shirai's observations, the methods and specimens were not the 
same to those used in their studies.

To examine this controversial hypothesis by the same method we analyzed naturally infected colonies of Leptotrombidium that were reared in our laboratory. However, IF often shows non-specific reactions especially in natural specimens, such as chigger mites. We therefore used a new IF procedure by using carbon tetrachloride to remove materials in the chigger mites that often produce the non-specific reactions (Urakami et al., 2000). Furthermore a battery of monoclonal antibodies specific to only one of the prototypes were used to increase the specificity to the antigens.

\section{Materials And Methods}

Chigger mites: Naturally infected colonies of $L$. arenicola (Traub) and L. fletcheri were provided by US Army Medical Research Unit, Kuala Lumpur, Malaysia and maintained in our laboratory (Takahashi et al., 1997). Each infected colony was derived from a single infected female. To maintain the colonies males were introduced to the colonies from uninfected colonies of the same species because males rarely occurred in the infected colonies (Rapmund et al., 1969, 1972; Takahashi et al., 1988). An infected colony of L. pallidum was established in our laboratory from mites collected in an endemic field in Japan and was maintained by brothersister mating, since both females and males emerged in the colony (Takahashi et al., 1988).

In vitro cultivation of $O$. tsutsugamushi: To isolate $O$. tsutsugamushi from each infected colony, a female adult was washed briefly in $80 \%$ ethanol for sterilization and homogenized in eagle's minimum essential medium (Nissui Pharmaceutical Co., Tokyo) containing $100 \mathrm{U} / \mathrm{ml}$ penicillin, $100 \mu \mathrm{g} / \mathrm{ml}$ streptomycin and $2 \%$ bovine serum, and inoculated onto a monolayer of cultivated mouse fibroblasts, L cells (Tamura et al., 1984).

O. tsutsugamushi adapted to L cells from L. fletcheri, L. arenicola and L. pallidum were designated as LF1, LA1 and LPT, respectively. We also cultivated five prototype strains, Gilliam, Karp, Kato, Kawasaki and Kuroki and used after plaque purification in the present study (Urakami et al., 1984). Suspensions of rickettsiae were prepared from homogenates of the infected L cells by differential centrifugation at $800 \times \mathrm{g}$ and $15,000 \times \mathrm{g}$ and smeared on slide glasses for IF observations.

Monoclonal antibodies and hyperimmune serum: Monoclonal antibodies against the TSA proteins of the five prototype strains were the same as those used in our previous report (Ohashi et al., 1996). To prepare hyperimmune sera females from inbred ddY mice colony (Japan SLC Inc., Tokyo) were serially infected with Gilliam, Karp and Kato strains at a monthly interval and later sacrificed to collect sera (Urakami et al., 2000). The resulting sera were highly reactive to all prototype strains including Kawasaki and Kuroki.

Immunofluorescent microscopy: For an analysis of the antigenicity of O. tsutsugamushi, embryos and adults in two or three generations were used in each species. Developing embryos were sandwiched between slide glasses to make smears on the both slides. Each adult mite was homogenized in $10 \mu 1$ of phosphate buffered saline (PBS) using an Eppendorf tubes and a piston, which was made by polymerizing epoxy resin in the tubes. Small amounts of the resulting homogenates were spotted on slide glasses. The following IF procedure was the same as mentioned previously (Urakami et al., 2000). Briefly, the smears on the slides were treated in carbon tetrachloride for $10 \mathrm{~min}$. The antigen spots were blocked by PBS containing $2 \%$ normal goat serum and reacted for $1 \mathrm{hr}$ at $37^{\circ} \mathrm{C}$ to a $1: 1,000$ dilution of the ascitic fluids containing the monoclonal antibodies. After washing in PBS the smears were then treated with fluorescein isothiocyanate-conjugated goat antimouse immunoglobulin (Cappel, NC. USA) diluted at $1: 1,000$ for $30 \mathrm{~min}$ at $37^{\circ} \mathrm{C}$. After washing in the same manner as above, the slides were mounted in $0.2 \%$ p-phenylenediamine (Sigma, MO. USA) in $100 \mathrm{mM}$ sodium bicarbonate, $\mathrm{pH}$ 9, and 50\% glycerol (Valnes and Brandtzaug, 1985) and observed with IB excitation in an Olympus BH5 microscope (Olympus Co., Tokyo). All smears that showed negative results in reaction with the monoclonal antibodies were washed in PBS and treated again with the hyperimmune serum diluted at $1: 1,000$ and the secondary antibody to confirm the presence of O. tsutsugamushi. When the smear was also negative in the latter reaction, indicating that the chigger mite had not been infected, the datum with the monoclonal antibody was omitted. This procedure was necessary because uninfected embryos frequently emerged in the colonies of L. arenicola and L. pallidum (Urakami et al., 2013).

To know the end-point titers of the monoclonal antibodies, the ascitic fluids containing monoclonal antibodies were serially diluted at three-fold and applied to the smears of O. tsutsugamushi in L cells and adult Leptotrombidium. Reactivity of the antibodies was expressed as the highest dilution that gave a positive reaction.

\section{RESULTS}

Antigenic types of O. tsutsugamushi in Leptotrombidium:

Table 1 shows the results of observations by using the monoclonal antibodies to know the antigenic types of O. tsutsugamushi in the embryos of the three species. We examined more than a dozen of embryos in each combination of the antibody and the mite species and calculated positive ratios. Each of the monoclonal antibodies diluted at 1:1,000 reacted with only one of the prototype strains, Gilliam, Karp, Kato, Kawasaki and Kuroki. O. tsutsugamushi in L. fletcheri reacted only to anti-Kato antibodies and those in L. arenicola and $L$. pallidum reacted only to anti-Karp antibodies. 
O. tsutsugamushi in L. fletcheri showed different reactivity to four anti-Kato antibodies. Positive reaction was revealed in all of 35 smears by 5D3 antibody, positive and weakly positive reactions were observed in 32 and 3 smears, respectively. However the other antibodies did not reveal positive reactions to all smears. 2C6, $3 \mathrm{~A} 6 \mathrm{E}$ reacted only to 38 out of $40(95.0 \%)$ and 4 out of 28 smears (14.3\%), respectively, and 1D12 showed negative reactions to all of 28 smears. Similar results were obtained from rickettsiae in $L$. arenicola and $L$. pallidum by the reactions of the anti-Karp antibodies. The examinations by antibody I- 19 were positive to all smears, but KP7 and 3C9 reacted to only some of the smears in both of the species.

End-point titers of the monoclonal antibodies to $O$. tsutsugamushi in chigger mites and cultured cells:

As shown in the above experiment, $O$. tsutsugamushi in the mites did not exhibit clear-cut reactivity to some of the monoclonal antibodies. To know the reason for the difference in the reaction, the precise reactivity

Table 1. Reactivity and positive ratios of monoclonal antibodies to O. tsutsugamushi in three species of Leptotrombidium.

\begin{tabular}{|c|c|c|c|c|c|c|c|c|c|c|c|}
\hline \multirow{3}{*}{ Mite species } & \multirow{3}{*}{$\begin{array}{c}\text { Reactivity of } \\
\text { antibody }\end{array}$} & \multicolumn{10}{|c|}{ Monoclonal antibodies against } \\
\hline & & \multicolumn{4}{|c|}{ Kato } & \multicolumn{3}{|c|}{ Karp } & \multirow{2}{*}{$\begin{array}{c}\text { Gilliam } \\
4 \mathrm{~B} 6\end{array}$} & \multirow{2}{*}{$\frac{\text { Kawasaki }}{417}$} & \multirow{2}{*}{$\frac{\text { Koroki }}{55-12}$} \\
\hline & & 5D3 & $2 \mathrm{C}-6$ & $3 \mathrm{~A} 6 \mathrm{E}$ & $1 \mathrm{D}-12$ & I-19 & KP7 & $3 \mathrm{C} 9$ & & & \\
\hline \multirow[t]{5}{*}{ L. fletcheri } & $++^{\mathrm{a}}$ & $91.4^{\mathrm{b}}$ & 80.0 & 10.7 & 0 & 0 & 0 & 0 & 0 & 0 & 0 \\
\hline & + & 8.6 & 15.0 & 3.6 & 0 & 0 & 0 & 0 & 0 & 0 & 0 \\
\hline & - & 0 & 5.0 & 85.7 & 100 & 100 & 100 & 100 & 100 & 100 & 100 \\
\hline & positive rate $e^{c}$ & 100 & 95.0 & 14.3 & 0 & 0 & 0 & 0 & 0 & 0 & 0 \\
\hline & total No. ${ }^{\mathrm{d}}$ & 35 & 40 & 28 & 28 & 29 & 20 & 20 & 28 & 29 & 27 \\
\hline \multirow[t]{5}{*}{ L. arenicola } & ++ & 0 & 0 & 0 & 0 & 100 & 28.9 & 11.4 & 0 & 0 & 0 \\
\hline & + & 0 & 0 & 0 & 0 & 0 & 5.3 & 25.7 & 0 & 0 & 0 \\
\hline & - & 100 & 100 & 100 & 100 & 0 & 65.8 & 62.9 & 100 & 100 & 100 \\
\hline & positive rate & 0 & 0 & 0 & 0 & 100 & 34.2 & 37.1 & 0 & 0 & 0 \\
\hline & total No. & 21 & 14 & 34 & 14 & 42 & 38 & 35 & 45 & 36 & 41 \\
\hline \multirow[t]{5}{*}{ L. pallidum } & ++ & 0 & 0 & 0 & 0 & 95.2 & 62.5 & 0.0 & 0 & 0 & 0 \\
\hline & + & 0 & 0 & 0 & 0 & 4.8 & 18.8 & 6.3 & 0 & 0 & 0 \\
\hline & - & 100 & 100 & 100 & 100 & 0.0 & 18.8 & 93.8 & 100 & 100 & 100 \\
\hline & positive rate & 0 & 0 & 0 & 0 & 100 & 81.2 & 6.2 & 0 & 0 & 0 \\
\hline & total No. & 13 & 15 & 14 & 14 & 21 & 32 & 32 & 16 & 19 & 15 \\
\hline
\end{tabular}

a: ++ : positive, + : weakly positive, - : negative. Antibodies were diluted at $1: 1,000$.

b: Percentage of smears that gave the reactivity of antibody indicated in left column.

c: Ratio (\%) of the number of positive and weakly positive to the total number examined.

d: The total number of embryos examined.

Table 2. End-titers of monoclonal antibodies to O. tsutsugamushi cultivated in L cells and in three species of Leptotrombidium.

\begin{tabular}{|c|c|c|c|c|c|c|c|c|c|c|c|}
\hline \multirow{3}{*}{ Antigen } & \multirow{3}{*}{$\begin{array}{l}\text { mite } \\
\text { No. }\end{array}$} & \multicolumn{10}{|c|}{ Monoclonal antibodies against } \\
\hline & & \multicolumn{4}{|c|}{ Kato } & \multicolumn{3}{|c|}{ Karp } & \multirow{2}{*}{$\begin{array}{c}\text { Gilliam } \\
4 \mathrm{~B} 6\end{array}$} & \multirow{2}{*}{$\frac{\text { Kawasaki }}{417}$} & \multirow{2}{*}{$\begin{array}{r}\text { Korok } \\
55-12\end{array}$} \\
\hline & & $5 \mathrm{D} 3$ & $2 \mathrm{C}-6$ & $3 \mathrm{~A} 6 \mathrm{E}$ & 1D-12 & I-19 & KP7 & $3 \mathrm{C} 9$ & & & \\
\hline \multicolumn{12}{|l|}{ L cells } \\
\hline Kato & & $>300 \mathrm{~K}^{\mathrm{a}}$ & $>300 \mathrm{~K}$ & $>300 \mathrm{~K}$ & $100 \mathrm{~K}$ & $<100$ & $<100$ & $<100$ & $<100$ & $<100$ & $<100$ \\
\hline Karp & & $<100$ & $<100$ & $<100$ & $<100$ & $>300 \mathrm{~K}$ & $100 \mathrm{~K}$ & $10 \mathrm{~K}$ & $<100$ & $<100$ & $<100$ \\
\hline LF1 & & $>300 \mathrm{~K}$ & $100 \mathrm{~K}$ & $1 \mathrm{~K}$ & $1 \mathrm{~K}$ & $<100$ & $<100$ & $<100$ & $<100$ & $<100$ & $<100$ \\
\hline LA1 & & $<100$ & $<100$ & $<100$ & $<100$ & $100 \mathrm{~K}$ & $3 \mathrm{~K}$ & $1 \mathrm{~K}$ & $<100$ & $<100$ & $<100$ \\
\hline LPT & & $<100$ & $<100$ & $<100$ & $<100$ & $>300 \mathrm{~K}$ & $30 \mathrm{~K}$ & $1 \mathrm{~K}$ & $<100$ & $<100$ & $<100$ \\
\hline \multirow[t]{3}{*}{ L. fletcheri } & 1 & $30 \mathrm{~K}$ & $3 \mathrm{~K}$ & 100 & 100 & $<100$ & $<100$ & $<100$ & $<100$ & $<100$ & $<100$ \\
\hline & 2 & $100 \mathrm{~K}$ & $10 \mathrm{~K}$ & 100 & 300 & $<100$ & $<100$ & $<100$ & $<100$ & $<100$ & $<100$ \\
\hline & 3 & $100 \mathrm{~K}$ & $10 \mathrm{~K}$ & 100 & 300 & $<100$ & $<100$ & $<100$ & $<100$ & $<100$ & $<100$ \\
\hline positive rate $(\%)^{\mathrm{b}}$ & & 100 & 95.0 & 14.3 & 0 & 0 & 0 & 0 & 0 & 0 & 0 \\
\hline \multirow[t]{3}{*}{ L. arenicola } & 1 & $<100$ & $<100$ & $<100$ & $<100$ & $100 \mathrm{~K}$ & 300 & 100 & $<100$ & $<100$ & $<100$ \\
\hline & 2 & $<100$ & $<100$ & $<100$ & $<100$ & $>300 \mathrm{~K}$ & 300 & 100 & $<100$ & $<100$ & $<100$ \\
\hline & 3 & $<100$ & $<100$ & $<100$ & $<100$ & $>300 \mathrm{~K}$ & $3 \mathrm{~K}$ & 300 & $<100$ & $<100$ & $<100$ \\
\hline positive rate (\%) & & 0 & 0 & 0 & 0 & 100 & 34.2 & 37.1 & 0 & 0 & 0 \\
\hline \multirow[t]{3}{*}{ L. pallidum } & 1 & $<100$ & $<100$ & $<100$ & $<100$ & $100 \mathrm{~K}$ & 300 & 100 & $<100$ & $<100$ & $<100$ \\
\hline & 2 & $<100$ & $<100$ & $<100$ & $<100$ & $>300 \mathrm{~K}$ & 300 & 100 & $<100$ & $<100$ & $<100$ \\
\hline & 3 & $<100$ & $<100$ & $<100$ & $<100$ & $>300 \mathrm{~K}$ & $3 \mathrm{~K}$ & 300 & $<100$ & $<100$ & $<100$ \\
\hline positive rate (\%) & & 0 & 0 & 0 & 0 & 100 & 81.2 & 6.2 & 0 & 0 & 0 \\
\hline
\end{tabular}

a: $\mathrm{K}$ indicates $\times 1,000$.

b: Ratio (\%) of the number of positive and weakly positive to the total number examined in Table 1. 
of these antibodies were determined by end-point titrations (Table 2). Smears of three individual adult females were used for all antibodies from each colony. Each monoclonal antibody used in the present study gave positive reactions at a dilution of $1: 10,000$ or higher against the strain used to immunize the mice when the antibodies had been generated (results by using smears of Gilliam, Kawasaki and Kuroki were not shown). The reactions however were negative even at a 1:100 dilution to the other four prototypes. The end-point titers to the smears of three individual adult females of the same species did not exceed 10-fold and some were as low as 1,000, the dilution used in Table 1 .

Antigenicity of the strains propagated in L cells, LF1, LA1 and LPT, was also examined by using the monoclonal antibodies and was compared with those in the mites. The overall reactivity of the antibodies to O. tsutsugamushi in the mammalian cells was the same to those in the mites. Many of the titers were, however, higher in the mammalian cells than in the mites.

\section{DisCUSSION}

It was previously indicated that the antigenic types of $O$. tsutsugamushi largely depended on the antigenicity of TSA proteins and could be further classified into several subtypes by reactivity of the monoclonal antibodies against the proteins (Ohashi et al., 1996, Tamura et al., 1997). Analyses of the TSA genes revealed that variety of the genes depended on not only the point mutations but also inter-strain shuffling of variable domains that composed the TSA genes (Ohashi et al., 1996). As a result the TSA proteins are significantly variable at various degrees among the strains, and that the antigenicity of TSA cannot be classified simply into several prototypes.

Shirai et al. (1979) developed a unique system for the antigenic typing of $O$. tsutsugamushi by using a battery of antibody mixtures prepared from rabbit hyper immune sera. Each mixture consisted of a fluorescence-conjugated antibody highly specific to one of prototypes and unconjugated antibodies specific to the other prototypes to block the heterogeneous reactions of the conjugated antibodies. By using a battery of such mixtures, each of which specifically stained only one of the prototypes, they found that many of $O$. tsutsugamush $i$ isolated from a single patient were stained with several mixtures. They obtained the same results in the analyses of chigger mites (Shirai et al., 1979, 1981 and 1982). They thus suggested that the individual mites and patients were infected simultaneously with several different antigenic strains. Their conclusions have been controversial in the serological studies of $O$. tsutsugamushi because of the difficulty to create a genetic theory to explain their observations. We herein designed our study to use the same methodology to Shirai et al. $(1979,1981,1982)$, but applied more sophisticated materials, monoclonal antibodies which had not been available when they studied.

The observations shown in Table 1 suggested that O. tsutsugamushi in each colony belonged to the same antigenical prototype, with a small difference in reactivity to the antibodies. It was, however, unclear whether the differences in the reactions between individual embryos, such as $2 \mathrm{C}-6$ and $3 \mathrm{~A} 6 \mathrm{E}$ to $L$. fletcheri, were caused by fluctuation in the antibody reaction or by variation of the antigenicity of $O$. tsutsugamushi in the individual mites as Shirai et al. $(1979,1981,1982)$ suggested. Clear correlations were observed between the positive rates and the end-point titers (Table 2), that is, the higher end-point titers were brought by the antibodies that had the higher positive rates. When $100 \%$ positive rate was yielded in Table 1, the end-point titers were 30,000 or higher, such as the reactions of 5D3 to L. fletcheri and I-19 to L. arenicola and L. pallidum. These observations strongly suggested that the ambiguous reactivity of the antibodies in the experiments of Table 1 could be caused by unstable reactivity of the antibodies near the end-points. It was therefore not necessary to explain the fluctuation of the reactivity of the antibodies by variation of the antigenicity of $O$. tsutsugamushi in Leptotrombidium. These results strongly suggested that at least three colonies used in the present study harbored only one antigenic type of O. tsutsugamushi. Though the antibody system by Shirai et al. $(1979,1981,1982)$ was well-designed, the discrepancy to our results may be caused by low specificity of polyclonal antibodies, sensitivity of immunofluorescent microscopy and difficulty in reduction of non-specific reactions in the mite tissues.

As shown in Table 2, O. tsutsugamushi that were adapted to the mammalian cells yielded higher endpoint titers than those in the mites. Rickettsiae in the mammalian cells were prepared by homogenization of the infected cells and centrifugal separations from the host cell materials. O. tsutsugamushi in the mites, however, was prepared from the mites without separation from components of the mite tissues. Such a difference in the preparations might result in different accessibility of the antibodies to the epitopes. O. tsutsugamushi is known to create fibrous structures in their cytoplasm that cannot be observed in mammalian cells in vitro (Wright et al., 1984), suggesting the differential gene expression depending on the hosts. This suggests the possibility that expression of the TSA genes may not the same in the mites and the cultured cells.

\section{ACKNOWLEDGEMENTS}

This study was supported in part by Grant-inAid from Ministry of Education, Science, Sports and Culture, and the Promotion (12670267) and Mutual Aid Corporation for Private Schools of Japan. 


\section{REFERENCES}

Elisberg, B. L., Campbell, J. M. and Bozeman, F. M. 1968. Antigenic diversity of Rickettsia tsutsugamushi: epidemiologic and ecologic significance. J. Hyg. Epidemiol. Microbiol. Immunol., 12: 18-25.

Murata, M., Yoshida, Y., Osono, M., Ohashi, N., Oyanagi, M., Urakami, H., Tamura, A., Nogami, S., Tanaka, H. and Kawamura, A. Jr. 1986. Production and characterization of monoclonal strain-specific antibodies against prototype strains of Rickettsia tsutsugamushi. Microbiol. Immunol., 30: 599-610.

Ohashi, N., Koyama, Y., Urakami, H., Fukuhara, M., Tamura, A., Kawamori, F., Yamamoto, S., Kasuya, S. and Yoshimura, K. 1996. Demonstration of antigenic and genotypic variation in Orientia tsutsugamushi which were isolated in Japan, and their classification into type and subtype. Microbiol. Immunol., 40: 627-638.

Rapmund, G., Upham, R. W. Jr., Kundin, W. D., Manikumaran, C. and Chan, T. C. 1969. Transovarial development of scrub typhus rickettsiae in a colony of vector mites. Trans. R. Soc. Trop. Med. Hyg., 63: 251-258.

Ree, H. I., Chang, W. H., Kee, S., Lee, I. Y. and Jeon, S. H. 1997. Detection of Orientia tsutsugamushi DNA in individual trombiculids using polymerase chain reaction in Korea. Med. Entomol. Zool., 48: 197-209.

Shirai, A., Dohany, A. L., Ram, S., Chiang, G. L. and Huxsoll, D. L. 1981. Serological classification of Rickettsia tsutsugamushi organisms found in chiggers (Acarina: Trombiculidae) collected in Peninsular Malaysia. Trans. R. Soc. Trop. Med. Hyg., 75: 580-582.

Shirai, A., Huxsoll, D. L., Dohany, A. L., Montrey, R. D., Werner, R. M. and Gan, E. 1982. Characterization of Rickettsia tsutsugamushi strains in two species of naturally infected, laboratory-reared chiggers. Am. J. Trop. Med. Hyg., 31: 395-402.

Shirai, A., Robinson, D. M., Brown, G. W., Gan, E. and Huxsoll, D. L. 1979. Antigenic analysis by direct immunofluorescence of 114 isolates of Rickettsia tsutsugamushi recovered from febrile patients in rural Malaysia. Jpn. J. Med. Sci. Biol., 32: 337-344.

Takahashi, M., Murata, M., Nogami, S., Hori, E., Kawamura, A. Jr. and Tanaka, H. 1988. Transovarial transmission of Rickettsia tsutsugamushi in Leptotrombidium pallidum successively reared in the laboratory. Jpn. J. Exp. Med., 58: 213-218.
Takahashi, M., Urakami, H., Yoshida, Y., Furuya, Y., Misumi, H., Hori, E., Kawamura, A. Jr. and Tanaka, H. 1997. Occurrence of high ratio of males after introduction of minocycline in a colony of Leptotrombidium fletcheri infected with Orientia tsutsugamushi. Eur. J. Epidemiol., 13: 79-86.

Tamura, A., Ohashi, N., Koyama, Y., Fukuhara, M., Kawamori, F., Otsuru, M., Wu, P.-F. and Lin, S.-Y. 1997. Characterization of Orientia tsutsugamushi isolated in Taiwan by immunofluorescence and restriction fragment length polymorphism analyses. FEMS Microbiol. Lett., 150: 225-231.

Tamura, A., Ohashi, N., Urakami, H., Takahashi, K. and Oyanagi, M. 1985. Analysis of polypeptide composition and antigenic components of Rickettsia tsutsugamushi by polyacrylamide gel electrophoresis and immunoblotting. Infect. Immun., 48: 671-675.

Tamura, A., Takahashi, K., Tsuruhara, T., Urakami, H., Miyamura, S., Sekikawa, H., Kenmotsu, M., Shibata, M., Abe, S. and Nezu, H. 1984. Isolation of Rickettsia tsutsugamushi antigenically different from Kato, Karp, and Gilliam strains from patients. Microbiol. Immunol., 28: 873-882.

Urakami, H., Okubo, K., Misumi, H., Fukuhara, M. and Takahashi, M. 2013. Transovarial transmission rates of Orientia tsutsugamushi in naturally infected Leptotrombidium colonies by immunofluorescent microscopy. Med. Entomol. Zool., 64: 43-46.

Urakami, H., Takahashi, M., Misumi, H., Okubo, K., Enatsu, T. and Tamura, A. 2000. Detection, isolation and characterization of Orientia tsutsugamushi in Leptotrombidium intermedium. Med. Entomol. Zool., 51: 1169-1177.

Urakami, H., Tsuruhara, T. and Tamura, A. 1984. Electron microscopic study on intracellular multiplication of Rickettsia tsutsugamushi in L cells. Microbiol. Immunol., 28: 1191-1201.

Valnes, K. and Brandtzaug, P. 1985. Retardation of immunofluorescence fading during microscopy. J. Histochem. Cytochem., 33: 755-761.

Wright, J. D., Hastriter, M. W. and Robinson, D. M. 1984. Observations on the ultrastructure and distribution of Rickettsia tsutsugamushi in naturally infected Leptotrombidium (Leptotrombidium) arenicola (Acari: Trombiculidae). J. Med. Entomol., 21: 17-27. 